\title{
A Sociocultural Perspective on Learning: Arabic And Indian Medical Students Compared
}

\author{
Viktoria. V. Zhura ${ }^{1}$, Yulia. V. Rudova ${ }^{1, *}$ \\ ${ }^{1}$ Volgograd State Medical University, Department of Foreign Languages with a Course of Latin, 400131, Volgograd, Russia
}

\begin{abstract}
The sociocultural backdrop of international students coming to a foreign country from different cultural and social environments plays a key role in shaping their attitude towards learning. The article explores and compares the motivational drives of international students from the Arab countries and India, which underlie their attitude to academic activities and academic progress. We used the method of systematic literature review, a questionnaire survey, comparative, quantitative, and qualitative analyses to obtain data on the value systems, traditions, and worldview of international students, which will make it possible for educators to appreciate the cultural contexts these students are embedded in. Social and cultural awareness of different aspects of the students' value systems will enable teachers in host countries to contribute effectively to international student integration into the educational process.
\end{abstract}

\section{Introduction}

International students arriving from a different cultural environment experience culture shock caused by new living conditions, new social ambience and cultural values, and new educational imperatives. One of the factors hindering the students' adaptation to the academic process is their sociocultural backdrop accounting for their attitude towards learning [1]. Therefore, cultural awareness of those aspects of the value system, traditions, and worldview of international students, which are immediately related to academic activities, will enable teachers to effectively integrate students into the educational process, which will ensure full coverage of the curriculum [2].

Moreover, the international students' belief systems encompassing their perceptions and attitudes to learning, expectations and emotional experience arise out of particular cultural contexts they are embedded in and their appreciation will make it possible for educators to develop empathy, become capable of extrapolating the values of other cultures on their native culture and constructing a joint identity within a global academic community [3].

The purpose of this study was to explore and compare the motivational drives of international students from the Arab countries and India underlying their attitude to academic activities and, finally, their academic progress.

The accomplishment of this goal involved the performance of a number of research activities described below:

1) a review and critical analysis of the experience gained by overseas teachers providing training to Arab and Indian students;
2) a survey of medical students of Volgograd State Medical University (Russia) coming from the Arab countries and the Indian subcontinent;

3) a comparative analysis of the culturally embedded education-related motivational drives of Arab and Indian students;

4) a comparative study of the findings of overseas research and the results of our investigation.

\section{Materials and methods}

To reach these objectives, the method of systematic literature review, a questionnaire survey, comparative, quantitative, and qualitative analyses were used.

\section{Results}

Arab students share similar beliefs based on the Islamic principles which may slightly vary due to the particular living conditions and local specificity, though. Following the Quran they have a high appreciation for such qualities as chasteness, perseverance, modesty, patience, kindness and generosity.

The Arabic system of education is based on the European model. The previous studies have demonstrated that a teacher in the countries of this region is regarded as an incontestable authority opening the door for moral and intellectual truths [4]. It is more important to guess what a teacher is driving at rather than searching for alternative wisdom. The most common teaching activity is the interaction when a teacher asks a question and a student gives an anticipated and "pre-planned" answer. As a rule, the ultimate goal of

\footnotetext{
* Corresponding author: juliarud@inbox.ru
} 
learning is to accumulate a finite and clearly cut set of knowledge and information. Moreover, no special emphasis is placed on such skills as analysis, synthesis and evaluation whereas memorization tends to be the most important learning approach.

The role model of a teacher anticipated by the students from this region involves activities when students write to the teacher's dictation and later the extent of the material acquisition is assessed. The assignments intended to be completed at home are left unattended as students expect their teacher to analyze it together with them in class [4].

In Arabic systems of education special emphasis is placed on the performance assessment through examinations. A zeal for being successful at the exams at all costs justifies the use of all possible kinds of cheating.

The recent study of the problems the American teachers have when training Arab students has reported that the latter strongly rely on memorizing and mere reproduction of information whereas they do not take much interest in independent research activities and creative thinking [4]. This accounts for the fact that both innovation and changes are not welcome by them. The most acceptable way of learning involves acquisition (memorization) of a limited amount of information provided in the textbooks recommended by the national education councils.

The gender aspect has also been discussed in a number of publications. It has been reported that male students demonstrate more freedom, vigour, assertiveness, and spontaneity. They tend to gain the initiative in a conversation and are reluctant to accept criticism [4]. We believe this aspect to be of great significance, however, it has not been elaborated in this study.

Another problem which claimed our attention was the Arab students' behavioral patterns associated with academic activities. Deception (cheating) is not considered immoral, as it "saves" the student's "face" [5]. In such communities one is not encouraged to openly admit making a mistake and thus commit a facethreatening act, therefore, such actions are avoided in every possible way.

The students from this region have liberal ideas of the nature and deadlines for submitting home assignments for check. The deadlines for submission are not restricted by the dates announced by the teacher and may be extended till tomorrow or even the end of the term.

Philosophical and axiological beliefs and ideas shared in the Arab communities underlie the students' perceptions of punctuality. Schedule delays and changes are quite common in their everyday life. There are not fixed working hours in many institutions and shops. Therefore, the time when a class is to start does not seem to be a matter of great concern and may be changed when circumstances require.

Not infrequently the students try to find a ready way to handle a problem at the sacrifice of hard work and maximum effort to achieve goals.
To optimize training strategies teachers must be well aware of culturally embedded interaction patterns of Arab students. They expect people to openly express their feelings and emotions; otherwise their behavior will be labeled as threatening and dishonest. Nonverbal behaviour is of great importance and also shows some distinction. A number of gestures used by Arab citizens cannot be readily and adequately interpreted by the members of other communities. Specifically, when giving a negative answer a student can move his head upward and click his tongue. It can also be an indication of disbelief or surprise. A beckoning gesture is used to say goodbye, whereas a pointing sign may pose a threat [6].

The idea of what personal space is felt as appropriate in the Arab countries also differs from that in European cultures.

By way of recapitulation, we sum up the conclusions of earlier studies exploring the issues of culturally embedded values of Arab students related to education. They are as follows: 1. Preference of passive acceptance of ideas over critical thinking. 2. Underestimation of the value of independent search for knowledge. 3 . Memorization and reproduction as the most important ways of intellectual activity; the goal of learning is to accumulate a certain amount of information. 4. An authoritative judgment prevails over to an independent judgment. 5. Individual ideas are not relevant and significant. 6. The expression of feelings and emotions is not acceptable in a classroom. 7. There is a single, unambiguous answer to any question $[4,7,8,9]$.

In contrast, India with its indigenous cultural identity characterized by ethnic/racial, linguistic and religious diversity offers an example of a country where rational westernized teaching ideas overlap with Eastern spirituality. Although the influence of Western philosophy on Indian education refers to the period of the British rule on the Indian subcontinent, there are contrasts across Indian education where cutting edge teaching technologies go together with traditional verbal teaching techniques, private schools stand in stark contrast to commonplace primitive schools, and secular educational establishments are worlds apart from religious schools.

A value-oriented approach toward sustainable development of a multifaceted personality encompassing his intellectual, social, esthetic, emotional, mental and religious dimensions underlies Indian teaching. According to Indian researchers, education is considered to be complete and comprehensive only if it aims to develop spiritual and moral values in a student [9].

Despite the low wages, the job of a teacher is one of the most respected and rewarding in India. Under the Indian education system, a teacher does not merely train or provide students with information; they try to shape students' attitudes, values, characters, etc. as exemplified by their own mode of living. Therefore, top qualities of a good teacher in India involve respect, tolerance, sympathy, honesty and a high level of cultural development. The teacher is considered as a friend, philosopher and guide [10]. 
There are significant differences in the way Indian students' performance is evaluated. Oral examinations are not used as a mode of assessing students' performance in India since the teacher is unable to question all students (60-70 students) in the class at a time. Written assessment tasks are used to uncover what has been learnt upon completing each topic and at the end of the semester.

Multiple changes in the world and specifically in Indian society resulting from scientific and technological advances, industrialization, IT revolution, liberalization and globalization make Indian students more creative, flexible within a changing environment, more ambitious in accomplishing their goals.

To briefly sum up, the concepts of modern Indian education are as follows:

1. Indian education seeks to develop the intellectual, moral, spiritual values and social awareness in students.

2. The aim of modern Indian education is to develop a multifaceted personality with inherent values and stereotypical behaviours.

3. The teacher in Indian education is a person who can help in a friendly way, give advice or explain in philosophical terms.

4. Written communication is more prevalent in Indian educational establishments than the oral one.

5. The changing environment makes Indian students adapt to new life conditions, thus encouraging them to acquire new qualities and traits.

The subsequent stage of our study involved a survey of Arab students from Egypt and Iraq (26 students) and students from India (26 students). The questionnaire ${ }^{a}$ covered questions pertaining to various aspects of the students' motivation, their ideas of academic activities, organizational and regulatory issues. Quantitative and qualitative analysis of the obtained data yielded a number of results which were later compared with earlier publications from abroad.

Our findings demonstrate that the main motivation factor during training was an ambition to become a highly qualified specialist (63\% Arab students and 53\% Indian students). A few students selected a combination of two answers - either "the wish to be a highly qualified specialist" and "my family responsibility", or "my family responsibility" and "curiosity to learn something new", which conforms to the earlier studies postulating family responsibility as an important motivation factor.

Another aspect which is crucial for uncovering the attitude of both Arab and Indian students towards learning is competitiveness among students. $72 \%$ of the respondents from Arab countries and $73 \%$ of Indian respondents regard competitiveness as a motivating factor, whereas $28 \%$ and $27 \%$, respectively, treat it as an antisocial factor hindering academic progress, which is also discouraging and annoying. These findings differ from the results of earlier studies which have reported about the negative assessment of competition in a classroom among Arab students. On the contrary, as previously mentioned, over the past decades, being

a The questionnaire included multiple-choice questions. ambitious about your goals has become an inherent quality in Indian students. We believe that the observed change in traditional beliefs is likely to result from gradual interiorization of the attitude to competition in higher education based on the values of the Russian culture as well as global education values.

A similar transformation was registered in the way they study for their classes at home. Instead of using the "small pain approach" described in previous studies, $54 \%$ of the respondents from the Arab countries and $46 \%$ of Indian students claimed that they try to do everything that is required, while $46 \%$ of Arab students do a little of everything as they do not have enough time to do all things. $36 \%$ of Indian students claimed that they prioritize only a few subjects when doing their home assignments.

Moreover, 56\% of Arab students believe that their curriculum contains subjects which are unnecessary and a waste of time. On the contrary, $44 \%$ of Arab and $50 \%$ of Indian students suppose that all the subjects may be useful in future, which conforms with the outcomes of the recent studies of Arab students insisting on the fact, that the acquisition of a selected, closed system of knowledge is considered most appropriate in these countries. Besides, $73 \%$ of Arab and only $46 \%$ of Indian students believe that the course information should use assignments to memorize important theoretical and factual information relevant to medicine just as $27 \%$ of Arab students chose such options as "assignments to learn to analyze things and make inferences" and "assignments to learn to find new solutions and justify them". This also agrees with the foreign studies of Arab students. It is noteworthy that the same number of Indian students $(46 \%)$ believed that the "assignments to learn to analyze things" were essential in teaching, thus proving the fact that the students expect to not only receive relevant information on the subject but also reflect it back using traditional methods of processing information. As few as $8 \%$ of Indian students chose the option "the course information should include assignments to learn to find new solutions and justify them". The fact that the majority of Indian students call for extending their knowledge in every possible way agrees with a widespread belief of Indian researchers that students in India are eager and willing to acquire a wide variety of new qualities in an attempt to improve themselves and adapt to the competitive environment [11].

A few distinctions have been discerned when analyzing the students' approach to learning. $37 \%$ of the respondents from Arab countries and $26 \%$ of students from India pointed out that they place the main emphasis on learning the information by rote, $36 \%$ of Arab students and $27 \%$ of students from India try to critically assess the information, whereas as few as $27 \%$ of Arab students and as many as $47 \%$ of Indian students suggest going beyond the scope of the information studied in class. We believe that the departure from traditional beliefs and transition to learning techniques (occurring at a different pace in Arab and Indian communities) is likely due to the accumulation of the new value 
orientations and adoption of transcultural educational values.

One of the integral parts of Russian as well as a number of overseas systems of education is the requirement for independent study of students outside a classroom labeled as homework. $47 \%$ of our Arab respondents believe that home assignments should be done together with a teacher without previous work at home. Still, only $44 \%$ of Arab students and as many as $66 \%$ of Indian respondents claim that home assignments should be done at home individually to revise the material. 9\% of Arab students think that they are not essential for study progress. These findings are similar to those of earlier studies of Arab students. Our findings on Indian students demonstrate that $30 \%$ of them claim that home assignments should be done together with a teacher without previous work at home, and the remaining $4 \%$ believe that home assignments are not essential for study progress. The results indicate that Indian students place a greater emphasis on self-control and individual student reflection, thus fostering their intellectual growth.

There are obvious distinctions pertaining to the terms of home assignment submission. As many as $81 \%$ of Arab students and $62 \%$ of students from India think that homework must be handed in on time, only $19 \%$ of Arab and $38 \%$ of Indian respondents believe that this may be done at the end of the term. The earlier mentioned process of gradual interiorization of the values of the host culture and the specific character of the education sphere account for these differences observed when previous studies are compared to.

The observed findings show significant distinction between our and overseas studies, which is likely to be due to the specificity of medical education, and its focus on the global standards of good medical practice, as well as greater flexibility of Arab students, which enables them to adjust to new environments.

Similarly to earlier studies, Arab students trained at Russian medical universities call for a finite and limited system of information. $83 \%$ of the Arab students expect their teacher to provide only essential information which makes it easier to be memorized. The remaining $17 \%$ want to be provided with a wider range of information to extend their knowledge of the subject. In case with the Indian students $74 \%$ of them want to be provided with a wider range of information to extend their knowledge of the subject, whereas the remaining $26 \%$ of Indian students expect to be provided with only essential information on the subject. These findings demonstrate that the Indian students are eager to meet a highly competitive and rapidly changing environment through learning beyond the scope of a discipline.

Practically the same proportion of the Arab respondents $(46 \%$ and $45 \%)$ anticipate their teacher to provide them with ready answers to exam and test assignments or find answers together with them, respectively. Just $9 \%$ stand for individual search for information. These findings are in congruence with the previous data and have revealed the approach to information search generally accepted in Arabic schools. In relation to the Indian student population the findings were as follows: $54 \%$ of Indian students chose the option "I want my teachers to find answers together with me without imposing them on me", $25 \%$ of Indian students want to search for information on their own, and the remaining $21 \%$ of the Indian respondents want to have ready answers to exam and test assignments. The fact that both Arab and Indian students (45\% and 54\%, respectively) anticipate their teacher to help them work out the answers to exam or test questions supports the general belief that the teacher is a guide who can help in a friendly way or/and give advice. As compared to Arab students, fewer Indian students $(46 \%$ and $21 \%$, respectively) want to have ready answers to exam and test assignments, thus proving the fact that Indian students stand for creative and critical thinking. This is also confirmed by the fact that $25 \%$ of Indian students want to be provided with the opportunity to work out answers on their own.

In contrast to the studies from abroad which reported the exams to be an important part of the academic process in the Arab countries, 55\% of the Arab respondents claim that they are useless as they just cause stress and nervousness. Only $27 \%$ of Arab and as many as $66 \%$ of Indian students believe that they are important as they help to critically assess their progress. $18 \%$ of Arabic and $34 \%$ of Indian students hope that exams help them to revise and systemize their knowledge. The culturally embedded differences in the ways of perceiving criticism (saving their positive and negative face) and critical evaluation may account for a significant discrepancy in assessing the value of exams and tests by these culturally diverse groups (Arabs and Indians) (27\% and 66\%, respectively). Along with this, the findings related to Indian students are consistent with the previous ones which indicated that in Indian culture a great emphasis is placed on revising and controlling their learning. Tests and exams are intended to assess a variety of mental/intellectual abilities, skills, qualities, etc. Therefore, Indian students argue for such a useful tool as exams and tests.

In addition, the marks their teachers give them must be based on how hard they tried and how regularly they attended classes $36 \%$ of Arab and $39 \%$ of Indian students) or may be used to encourage them to work harder $(38 \%$ of Arab respondents and $61 \%$ of Indian ones). However, $26 \%$ of Arab respondents do not place any value on them. Our findings demonstrate that Indian students, who tend to be more competitive, seek more encouragement from their teachers than Arab students.

Similarly to earlier studies, our results demonstrate that the ideas of the Arab students of the regulatory aspect of the academic life are culturally embedded. $73 \%$ of Arab and only $43 \%$ of Indian students think that discipline requirements should not be strict, i.e. the class schedules, break schedules can be changed by a student if any personal problems arise. However, the remaining 27\% and $57 \%$, respectively, believe that they must be precisely followed. A greater emphasis on observing disciplinary requirements by a higher proportion of Indian students has demonstrated that Indian students tend to adhere to the principles of punctuality, 
attendance and commitment to learning as compared to the Arab community.

The majority of Arab respondents (56\%) and $51 \%$ of Indian students regard their teacher as a person who must help them with everything. $25 \%$ of Arab and $49 \%$ of Indian students consider the teacher to be an incontestable authority, whereas $9 \%$ of the respondents from the Arab countries look upon the teacher as a partner with whom they can argue and disagree. Our findings concur with the conclusions made by Singh and Nath [11] of the teacher being a friend, philosopher and guide, who can help, give advice and/or explain if required.

\section{Discussion}

A comparative study of earlier findings and our empirical data has yielded a number of speculations, which are presented below.

The analysis of motivational drives and sociocultural expectations of Arab students has demonstrated that the organization of their education in host countries has to encompass the measures to develop their motivation and adjust their approach to learning so that its specific objectives could be fully accomplished. Sociocultural expectations of the Indian students require a competitive environment in a classroom with a sufficient amount of assigned tasks.

First of all, the sociocultural value of the teacher's role has to be revised. The fact that he is thought about as the central link of the academic process, who is responsible for quantifying and qualifying knowledge, actively involved in both the acquisition of information in a classroom and at home cannot be neglected. Its underestimation may cause students' frustration and dissatisfaction. Similarly, in Indian culture teachers are revered by students as philosophers, guides, role models, those whom parents entrust to shape their children's characters, values, etc. However, the process of developing students' autonomy and ability for independent study must be continuous and stepwise.

When selecting course materials an optimal combination of reproductive activities (requiring memorization and replication of information) and productive activities (requiring individual independent search) is essential. Moreover, the teacher has to demonstrate how the latter will promote students' expertise and academic progress.

There is an urgent need for exemplifying interdisciplinary relationships subsistent in practice during theoretical training. This will extend the students' interest in various subjects and urge them to go beyond the limits of the basic set of information. Furthermore, practical examples, problem-solving activities, and case studies can be used to demonstrate the importance of critical analysis approach to operating available information.

Great effort is required to enhance discipline awareness and communication standards. Students have to be informed about the principles of cooperation in communication, potential aftermaths of ignoring discipline requirements at work in their future professional activities, which will also contribute to adjusting some of their culturally embedded beliefs and values to the standards of their subsequent clinical activities.

The survey highlights the importance of encouraging intense competition [12], the drive for a successful career, enthusiasm and willingness to study hard among Indian students. This necessitates the use of innovative teaching methods including IT technology and their regular update, which concurs with their culturally embedded beliefs $[13,14]$ about a value-oriented approach. Focus should be placed on shaping a multifaceted personality by not only providing the course material, but also by highlighting the importance of revision, autonomy, students' individual search, memorization, recollection, improving students' communication skills, etc.

\section{Conclusion}

The present findings suggest that today's educators working in the global world have to develop cross cultural awareness encompassing the knowledge of the international students' cultural values and expectations related to training, the ways of balancing and converging them with the beliefs the receiving system of education rests on, and the world-wide views inherent in a particular professional field. This will help to build transcultural awareness and worldview, which is of huge importance for occupational spheres requiring continuous professional migration, exchange of both information and human resources, and the ability to interact with clients belonging to different cultures.

\section{References}

1. G. Allport Attitudes and opinions (Lawrence Erlbaum Associates Publishers, London, 2004)

2. Z. F. Beykont The power of culture: Teaching across language difference (Harvard Education Publishing Group, Cambridge, 2002)

3. K. A. Appiah The ethics of identity (Princeton University Press, New Haven, 2005)

4. A. Helms Handbook for ESL teachers of Arab Students Retrieved from: https://sfsudspace.calstate.edu/bitstream/handle/10211.3/139765 /AS361984A336.pdf? sequence=1 (1984)

5. P. Brown, S. Levinson Politeness: some universals in language usage (Cambridge University Press, Cambridge, 2014)

6. R. A. Barakat, JPC, Arabic Gestures, VI (4), 749-787 (1973)

7. A. Benahnia, ESJ, Application of Motivation in Arab EFL/ESP Learners' settings: A Socio-cultural Approach, 13. 4, 73-86 (2017)

8. S. P. Chamberlain, ISC, Recognizing and responding to cultural differences in the education of culturally and linguistically diverse learners, 40, 195-211 (2005) 
9. G. I. Sadek, Arab Students' Attitudes toward western Culture and Motivation to Learn English Retrieved from:

https://dspace.aus.edu:8443/xmlui/bitstream/handle/1 $1073 / 41 / 29.232-$

2007.02\%20Gihane\%20Sadek.pdf?sequence=1\&isAl lowed=y (2007)

10. A. Pathania, Academé, Teachers role in Quality Enhancement and Value Education, 24(1), 19-25 (2011)
11. Y.K. Singh, R. Nath, Education in emerging Indian society (New Delhi, APH Publishing Corporation, 2005)

12.P. Roshan, P. Poonam, J.S. Devendra, IJCMAS, Decline of Ethics and Moral Values in Present Scenario - An Analysis, 7 09, 1085-1092 (2018)

13. D.S. Ackerman, B. L. Gross, JME, Instructor Feedback: How Much Do Students Really Want?, 32, 2, 172-181 (2010)

14. S. Bhagat, IJIP, Ethical Indian education system - a need of the nation, 3, 2, 7, 100-104 (2016) 